\title{
A Review on Capacity Optimization of Hybrid Renewable Power System with Energy Storage
}

\author{
Jiatian Gan ${ }^{1}$, Jingli $\mathrm{Li}^{2}$, Wannian $\mathrm{Qi}^{2}$, Aynur $\mathrm{Kurban}^{3}, \mathrm{Yi} \mathrm{He}^{3}$ and $\mathrm{Su} \mathrm{Guo}^{3, *}$ \\ ${ }^{1}$ Qinghai Electric Power Research Institute, Qinghai, China \\ ${ }^{2}$ Qinghai Golmud Luneng Energy Co., Ltd (Ducheng Weiye Group Co. Ltd), Qinghai , China \\ ${ }^{3}$ College of Energy and Electrical Engineering, Hohai University, Nanjing, China
}

\begin{abstract}
With the rapid development of the global economy, there is a sharp shortage of fossil energy sources. Therefore the development of renewable energy technologies such as wind power and solar power has become a hot issue nowadays. However, due to the randomness, intermittency and instability of renewable energy, it is difficult to provide continuous and stable electricity when it runs independently. Hybrid renewable power systems with energy storage can improve the reliability of power supply. Capacity optimization is the key of hybrid renewable power system design and the basis of optimal scheduling. In this paper, the capacity optimization of hybrid renewable power system with energy storage is summarized and classified. According to the different energy storage modes of the hybrid renewable power systems, the capacity optimization models, optimization methods and the software used are introduced.
\end{abstract}

\section{Introduction}

With the sharp increase of energy demand, the rapid consumption of fossil energy and the social attention of the environment protection, the utilization of renewable energy is rising rapidly around the world. However, due to the constraints of intermittent and random characteristics of renewable resources, the renewable energy power systems, such as solar energy, wind energy, cannot be continuously and steadily powered, which have negative impacts on the stability and reliability of power system operation.

In recent years, making full use of the complementarity characteristics of various renewable resources, and combining energy storage, hybrid renewable power systems have been proposed to achieve stable power output and reduce the impacts on the power grid. The existing researches mainly focus on wind power-photovoltaic system (WT-PV system) [1-3], photovoltaic-concentrated solar power system (PV-CSP system) [4-6], WT-PV-hydropower system [7], WT-PV storage system [8-10], and so on.

Capacity optimization is the key of hybrid renewable power system design and the basis of optimal scheduling. Reliable and stable power supply can be achieved, and the energy utilization rate and economic performance of the system can be improved through the capacity optimization. Therefore, it is necessary to optimize the capacity configuration of hybrid renewable power systems.

In this paper, the classification of capacity optimization of hybrid renewable power systems with energy storage is summarized in Section 2. Optimization algorithms are introduced in Section 3. Application software are summarized in Section 4. Finally, the conclusions are presented in Section 5.

\section{Classification of capacity optimization of hybrid renewable power systems}

\subsection{According to grid-connected and off-grid}

There are many types of models for capacity optimization of hybrid renewable power systems. According to whether it is connected to the grid, it can be divided into grid-connected type $[9,10]$ and off-grid type [11]. The capacity optimization of grid-connected hybrid renewable power system is mostly based on the returns on investment (ROI) as the optimization objective, while that of the off-grid hybrid renewable power system takes the reliability and stability of power supply as the optimization objective. Xu et al. [12] considered the capacity optimization of wind-solar hybrid renewable power system under both stand-alone and grid-connected. $\mathrm{He}$ used the wind-solar complementary characteristics to reduce the battery capacity and the frequency of charges and discharges when off-grid, and adopted the time-sharing optimization strategy to meet the power fluctuation constraints when grid-connected.

\subsection{According to energy storage forms}

Renewable energy sources such as solar and wind energy will be great affected by weather and seasons. Energy

\footnotetext{
* Corresponding author: guosu81@126.com
} 
storage system is an effective means to improve energy utilization and suppress power fluctuation. Therefore, Energy storage is an important part of hybrid renewable power systems [13]. According to the different ways of energy storage, hybrid renewable power systems can also be divided into many kinds. Annette Evans et al. [14] studied the energy storage modes of renewable energy, introduced and compared three common energy storage modes of thermal energy storage (TES), pumped hydro storage, and battery storage (BS). Among them, TES has low cost and large capacity, which is suitable for peak load shifting; pumped hydro storage has low investment risk, low power generation cost, but high construction cost, low energy density, which is suitable for large-scale construction; BS has fast response, high energy density and no limitation of natural conditions, but high cost. When it is applied with energy storage system in hybrid renewable power system, the optimal energy storage mode can be selected according to demand, cost, efficiency and other factors. A classification of capacity optimization of the hybrid renewable power system is as follows, according to different energy storage methods.

Batteries are widely used in multi-energy complementary generation and power grid operation assistance because of their relatively mature technology. Wang et al. [15] proposed an optimal capacity configuration strategy of wind-storage hybrid power system, which used sodium-sulfur batteries with faster response as energy storage units, based on the characteristics of wind power and operation constraints of BS system to suppress power fluctuations in the power grid and improve the economy of the system. Orhan Ekran et al. [3] introduced a capacity optimization method of response surface methodology for a WT-PV hybrid power system with BS shown in Fig.1. Liu et al. [16] combined constraint functions, battery charge \& discharge constraints and power supply number constraints to propose a multi-objective function with the minimum total cost of the system, and the minimum values of loss of power supply probability (LPSP) and surplus of power supply probability (SPSP). Ding et al. [17] studied the economy of an independent micro-grid system. Considering the scheduling strategy of the system, an optimization model was established with the objective function of minimizing the overall cost.

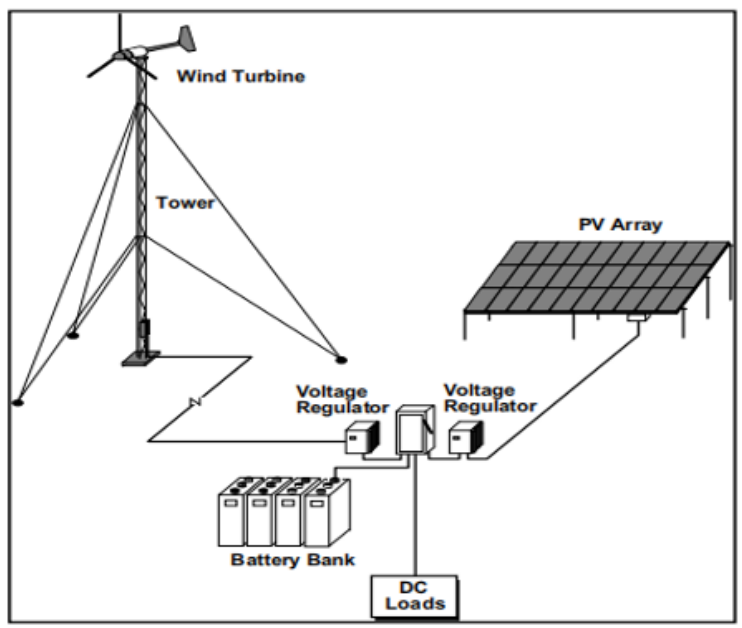

Fig.1 Schematic diagram of a WT/PV/BS hybrid energy system [3]

Although batteries have many advantages, they have short cycle life, high price and strict charging and discharging restrictions. The hybrid energy storage mode of battery and super capacitor can reduce the cost and enhance the output performance of the system. Yu et al. [18] make use of the complementary characteristics of super capacitors and batteries to apply hybrid energy storage in grid-connected wind-solar complementary power generation system. Firstly, primary capacity optimization is calculated with the objective function of the minimum system cost. Then genetic algorithm is used to further optimize the hybrid energy storage system of battery and super capacitor, so as to obtain the optimal system capacity. Shen et al. [19] studied the capacity optimization method of the wind-solar energy storage system with battery and super capacitor. It has analyzed the principle of each part of the wind-solar energy storage system, the advantages of hybrid storage system of battery and super capacitor, and proposed a capacity optimization model with the reliability indexes of SPSP and LPSP.

The pumped hydro storage is the most mature and commercial technology of energy storage. Wu et al. [7] aimed at minimizing life-cycle cost, established optimization model with water balance and power reliability as constraints. The results show that the WTPV-hydropower hybrid power system can guarantee high reliability and stability of power supply when running independently; when running in grid, it can reduce economic cost and improve power supply reliability. Zhu et al. [20] optimized the capacity of the hydro-PV-WT complementary power system under certain conditions of curtailment of wind and solar, considering the objective functions of minimum curtailment of wind power and solar power and maximum total installed capacity of WT and PV, combining with the constraints of system channel capacity, certain wind and solar irradiation curtailment rate and output constraints of hydropower station. A wind-pumped hybrid energy system with energy recovery hydraulic system is shown in Fig. 2 .

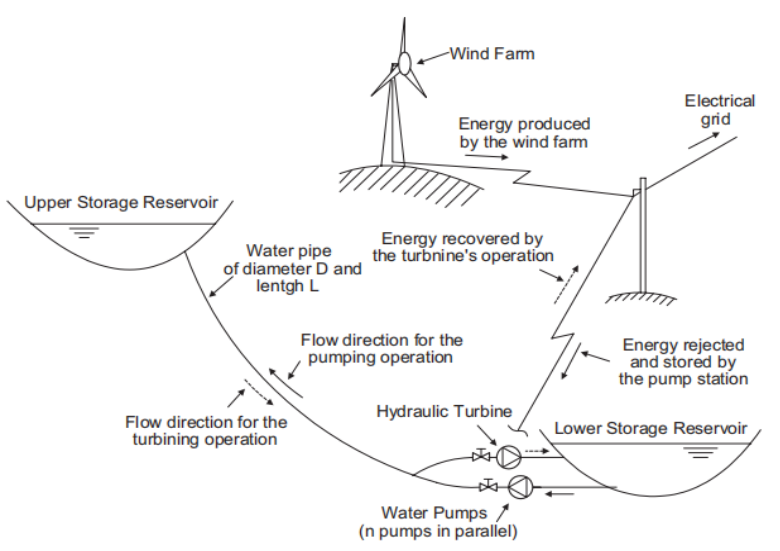

Fig.2 Schematic diagram of a wind- pumped hybrid energy system [21] 
The thermal energy storage system (TES) is cheap and easy for large scale applications and can play a role of peak load shifting. For example, Zhai et al. [22] proposed a PV-CSP hybrid power system with large capacity and low cost TES (shown in Fig. 3), which replaces expensive batteries, and stores excess energy of PV plants with TES by electric heater. Therefore, it is very suitable to be applied to renewable energy for power generation. Yang et al. [23] also introduced electric heater in a hybrid power system which includes a CSP plant and a wind farm to reduce wind curtailment. Electric heater can convert excess wind energy into heat energy stored in the TES of CSP plants, which can be used to generate electricity when it needed. This can not only reduce energy loss, but also improve the reliability of power supply. The optimal dispatch of the combined generation system is a mixed integer linear programming (MILP) problem, while the optimal objective is maximize profit. Adriana et al. [5] studied the PV-CSP hybrid power system with BS and TES. It provided the basic load using the molten salt TES of the solar power tower and the BS of the PV station. The photovoltaic scale, the TES capacity and the battery size were analyzed and was studied according to the capacity factor and LCOE.

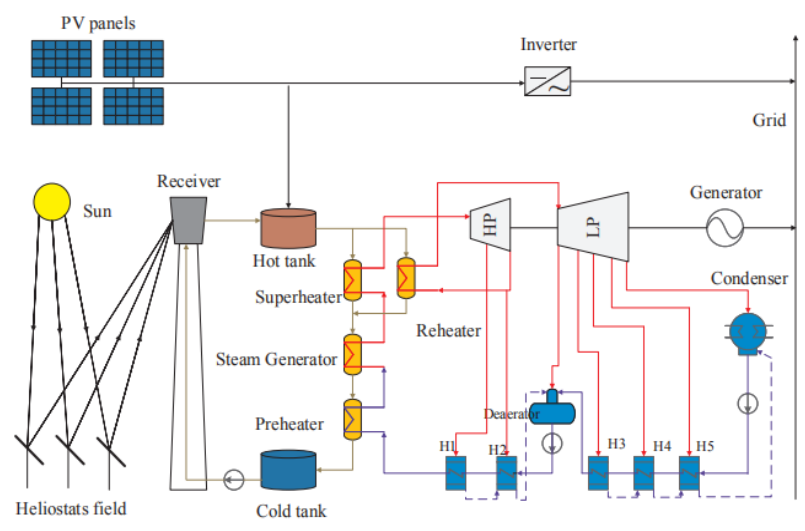

Fig.3 Schematic diagram of a thermal storage PV-CSP system [22]

\section{Optimization methods}

Al-Falahi et al. [24] studied three capacity optimization methods: classical algorithm, modern technology and software tools. It is pointed out that in solving complex optimization problems, modern technology based on artificial intelligence algorithm (AI) is more flexible and practical than classical algorithms, and hybrid algorithms can get more ideal optimization results than single algorithms. Modern algorithms of capacity optimization of hybrid renewable power system can be summarized to artificial neural network algorithm (ANN), swarm intelligence algorithm, and so on. Swarm intelligence algorithm includes genetic algorithm (GA), particle swarm optimization (PSO), ant colony algorithm (ACO), simulated annealing algorithm (SA), crowd search algorithm (CSA) and many other algorithms. Among them, the most common optimization algorithms are GA and PSO. GA has a mature analysis method in convergence, and the convergence speed can be estimated. PSO is a fast algorithm, but its shortcoming is that it is easy to fall into local optimization and its search accuracy is not high. Liu [16] uses the improved GA to solve the multi-objective optimization problem. By improving the fitness function of GA, the slow convergence speed of the conventional GA is solved. Wu et al. [7] and Ding et al. [17] used GA to solve the model, and obtained the optimal capacity of each power supply. Chen et al. [25] proposed a multi-objective function considering the lowest cost of the system and LPSP, which was solved by an improved multi-objective composite differential evolution (DE) algorithm. This algorithm introduces the concepts of congestion degree and Pareto sorting into the DE algorithm and has the ability of multi-objective optimization. In addition, the chaotic PSO algorithm is applied to the optimization problem to solve the maximum power point tracking problem of photovoltaic arrays under local shadows. Yang et al. [26] improved the PSO algorithm by optimizing the asymmetric acceleration factor. The simulation results show that the method accelerates the convergence speed and optimizes the working state. However, there are still some problems such as easy to fall into local optimum and low search accuracy. Mao et al. [27] proposed a MILP problem for self-generation and load scheduling models considering carbon emissions and time-of-use tariffs in order to obtain the best economic and environmental benefits. The carbon trading cost and electricity cost is set as the optimization goal of the model. Orhan Ekran et al. [3] put forward a response surface methodology for capacity optimization of WT-PV combined power generation system.

\section{Application software and language}

As for application software used in the solution of capacity optimization of hybrid renewable power system, Mao et al. [27] proposed a MILP problem solved by CPLEX. The results show that the model is more economical and environmental friendly than the conventional model and can effectively reduce the total cost of the system and carbon emissions. Hu et al. [11] studied the grid-connected WT-PV hybrid power system. Aiming at the total revenue in the whole life cycle, the capacity optimization model was established under the constraints of wind and solar energy utilization ratio, WT-PV complementarity characteristics, and tracking dispatching curve. Hybrid Optimization Model for Electric Renewables (HOMER) software was developed by National Renewable Energy Laboratory (NREL) USA. With the help of HOMER Software, the monthly average wind speed and solar radiation data are discretized into hourly average data as data sources of wind and solar resources. A variable step cycle discrete method was used to solve the model. Yu et al. [18] also used the HOMER simulation software to optimize the system capacity to obtain the optimal solution. The randomness, ergodicity and regularity of GA are combined with Matlab simulation software to complete 
the calculation of the hybrid power system optimization model. Anurag Chauhan [28] introduced many applications for integrated renewable energy system. Among them, Hybrid Optimization by Genetic Algorithm (HOGA) software is used to output multiobjective optimization, energy supply analysis according to constraints resource, component data. HOGA was developed by Electric Engineering Department of the University of Zaragoza (Spain).

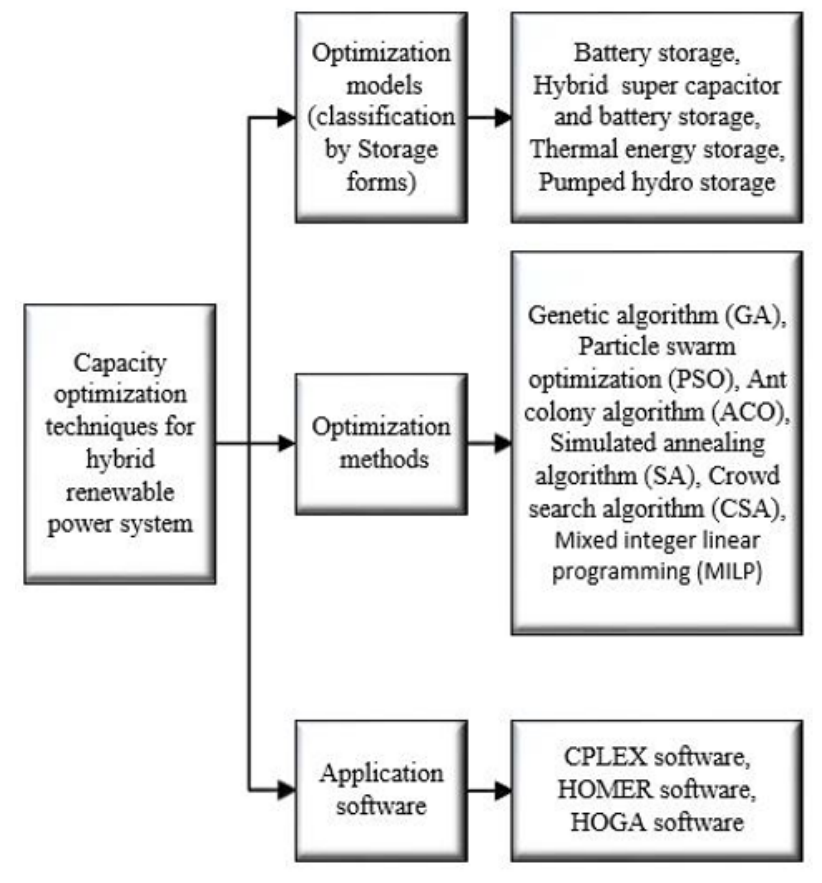

Fig.4 Capacity optimization techniques

\section{Conclusion}

The capacity optimization of hybrid renewable power system is summarized in this paper by classification according to the storage forms, optimization algorithm, and application software. Economy and power supply reliability and stability are commonly used as optimization objectives. Energy storage plays an important role in hybrid renewable power system. Among several energy storage forms in this paper, BS has high response speed and large energy density; hybrid energy storage of BS and super capacitor can effectively improve the output performance of the system; pumped hydro storage has low investment risk, low power generation cost, but high construction cost; TESS has large capacity and low price, and is easy for large-scale construction. Improved optimization algorithms, such as improved GA and PSO can solve the problems of slow convergence and easy to fall into local optimum. Finally, the application of HOMER software, CPLEX software and HOGA software in the capacity optimization model of hybrid renewable power system is introduced.

Financial support by State Grid Corporation of China Science and Technology Projects (SQQHKY0DWJS1800170) and Qinghai Golmud Luneng Energy Co., Ltd Projects "Research on key technologies of Wind-Solar-CSP-Battery storage control system based on Haixi multi-energy complementary integration and optimization demonstration project", is very much appreciated.

\section{References}

1. Anoune, K.,Bouya, M.,Astito, Abdelali, Abdellah, A. Renewable and Sustainable Energy Reviews 93(2018) 652-673

2. W. Hongbin, C. Bin, G. Caiyun. Journal of Agricultural Engineering 27(2011) 241-245

3. O. Ekren, B. Y. Ekren. Appl. Energy 85 (2008) 1086-1101

4. Ju, X. ,Xu, C.,Hu, Y.,Han, X. Wei, G.,Du, Xiaoze. Sol. Energy Materials and Solar Cells 161 (2017) 305-327

5. A. Zurita, C. Mata-Torres, C. Valenzuela, C. Felbol, J. M. Cardemil, A. M. Guzmán, R. A. Escobar. Solar Energy 173 (2018) 1262-1277

6. Starke, Allan R.Cardemil, José M.Escobar, Rodrigo, Colle, Sergio. Energy 147(2018) 490-503

7. W. Wanlu, W. Gang, X. Lirong, Z. Hao. Power and energy 35 (2014) 88-92

8. D. Tianyi, L. Wenyi, C. Bingqing, T. Hao. Solar Energy 39 (2018) 2439-2445

9. Y. Fan. Research on capacity optimization deployment of off grid wind solar storage and complementary generation system. Tianjin University 2012

10. D. Aihua. Application of Energy Technology (2018) 47-52

11. H. Linxian, G. Yayun, Y. Yousu. Power grid and clean energy 32 (2016) 120-126

12. X. Lin, R. Xinbo, Z. Buhan, M. Chengxiong. Chin.J.Elec.Eng.32 (2012) 88-98+14

13. YANG H,LU L,ZHOU W. Solar Energy 81 (2007) 76-84

14. A. Evans, V. Strezov, T. J. Evans. Renewable and Sustainable Energy Reviews 16 (2012) 4141-4147

15. W. Xiaolei, G. Jia, Z. Jiawei. Zhejiang Electric Power 37 (2018) 14-17

16. L. Yanping. Capacity optimization and coordinated control method for wind-solar hybrid power generation system. Shandong University, 2017

17. D. Ming, W. Bo, Z. Bo, C. Power grid technology 37 (2013) 575-581

18. Y. Dongxia, Z. Jianhua, W. Xiaoyan, Gaoyuan. Journal of Power Systems and Automation 1-8

19. S. Xijun. Research on control strategy and energy storage capacity optimization of wind-solar complementary power generation system. Shanxi University (2014)

20. Z. Yanmei, C. Shijun, H. Weibin, W. Li, M. Guangwen. Hydropower Energy Science 36 (2018) 215-218 
21. J.S. Anagnostopoulos, D.E. Papantonis. Renewable Energy 33 (2008) 1685-1694

22. R. Zhai, H. Liu, Y. Chen, H. Wu, Y. Yang. Energy Conversion and Management 154 (2017) 56-67

23. Y. Yang, S. Guo, D. Liu, R. Li, Y. Chu. Energy Conversion and Management 160 (2018) 243-250

24. Al-falahi, Monaaf D. A. Jayasinghe, S. D. G. Enshaei, H. Energy Conversion and Management143 (2017) 252-274

25. C. Tian. South China University of Technology,(2017)

26. Y. Guohua, Z. Xiangfen, M. Yujuan, H. Shijun, W. Jinmei, W. Pengzhen. Electrical measurement and instrumentation 52 (2015) 1-5+10

27. M. Tan, Y. Chen, Y.-x. Su, S.-h. Li, H. Li. Journal of Cleaner Production (2018)

28. Chauhan, Anurag. Saini, R.P. Renewable and Sustainable Energy Reviews 38 (2014) 99-120 